\title{
POLYLACTIC ACID INTERACTIONS WITH BIOCERAMIC SURFACES
}

\author{
IZABELLA IRSAI ${ }^{\mathrm{a}}$, ADRIAN M.V. BRÂNZANIC ${ }^{\mathrm{b}}$, \\ RADU SILAGHI-DUMITRESCUa, ${ }^{a}$
}

\begin{abstract}
Molecular dynamics simulations were employed in order to analyze the interfacial interaction of polylactic acids with zirconia and hydroxyapatite surfaces. The interactions of polymers on five crystallographic planes were simulated. Silane coupling agents can improve the interactions between the bioceramic surfaces and the polylactic acids. The effects of the coupling agents are more evident in the presence of hydroxyapatite surfaces. Weak interactions hold together the polylactic acids and bioceramic systems. These interactions are formed between the hydrogen atoms from methyl groups or from the main chains of the polylactic acids and the oxygens of the surfaces. Polylactic acids change their conformations after molecular dynamics simulations due to the interactions. The conformation changes are more obvious when silane coupling agents are added to the polylactic acids and bioceramic systems.
\end{abstract}

Keywords: polylactic acid, hydroxyapatite, zirconia, molecular dynamics, weak interactions

\section{INTRODUCTION}

Biomaterials are biocompatible and non-toxic materials that are not recognized by the body as a potentially harmful foreign substances. They are classified as bioinert, resorbable and bioactive according to tissue response. Bioinert materials induce formation of a fibrous tissue of variable thickness, interfacial bond forms on bioactive materials and resorbable materials are replaced by the surrounding tissues [1]. Biomaterials belong to all 5 major

a Babeş-Bolyai University, Faculty of Chemistry and Chemical Engineering, 11 Arany Janos str., RO-400028, Cluj-Napoca, Romania

b Babeş-Bolyai University, Institute of Interdisciplinary Research in Bio-Nano-Sciences, 42 Treboniu Laurian str., RO- 400271, Cluj-Napoca, Romania

* Corresponding author: radu.silaghi@ubbcluj.ro 
classes of materials: metals, ceramics, polymers, composites and natural materials. Bioceramics are a class of materials that satisfy most of these criteria in addition to their biocompatibility and sufficient mechanical strength close to that of bone. The bioceramics have low density, high hardness, low tensile strength and high compressive strength and they are characterized by chemical stability. Bioceramics induce a specific biological response at the interface of the material resulting in the formation of strong bond between the tissue and the material.

Hydroxyapatite (HA) is a highly biocompatible ceramic that is characteristically resorbable in body. In practice, hydroxyapatite is either used as a bioactive coating on implants, or reinforced by metal or ceramic phases. Zirconia is an inert ceramic in its pure form. The properties of interest to the engineer utilizing zirconia ceramics include strength, toughness, hardness, wear resistance and thermal properties $[2,3]$.

Most polymers, especially thermoplastics, are non-polar (hydrophobic) substances, which are not compatible with polar (hydrophilic) surfaces, poor adhesion can result between polymer and material. To improve the adhesion between surfaces and polymers coupling agents have been employed [4-7]. Coupling agents are used in small quantities to treat a surface so that bonding occurs in the system. Bonding agents act as bridges that link the material and the polymer. Coupling agents are classified into organic (e.g. isocyanates, anhydrides, amides, imides), inorganic (e.g. silicates), and organic-inorganic groups (e.g. silanes). Silanes, represented as R-Si(OR') $)_{3}$, have better performance in organic-inorganic coupling agents, because the attachment of silanes to hydroxy groups of cellulose or lignin is accomplished either directly to the alkoxy group (-OR') attached to silicon or via its hydrolyzed products (i.e. silanol) by the hydrogen bonds or ether linkage [8]. The functional group $(R)$ in silanes also influences the coupling action [9-15].

Hydroxyapatite/polymer composites improve the HA mechanical properties.[16-18] HA polymer composites present good bioactivity due to the HA content [19]. The most intensively studied biopolymers are polyethylene [20, 21], polyamide [22, 23] and polylactic acid (PLA) [24-26]. These polymers are used as matrices in $\mathrm{HA} /$ polymer composites. The polyethylene/HA composites are used as hard tissue replacement. The chemical structure of polyamide is similar to the collagen structure; therefore polyamide can be utilized as a composite matrix. Polylactic acid /HA composites are employed in degradable internal bone fixation devices.

Reported here is a computational exploration of polylactic acid interactions with solid surfaces via non-covalent interactions, aiming to identify modes of binding and binding strengths. 


\section{RESULTS AND DISCUSSION}

The interaction energies of polylactic acid/zirconia and PLA/silane/ zirconia systems are listed in Table 1 . The negative values indicate that in all cases binding of the different types of PLA to the zirconia surface is favorable energetically.

Table 1. The interaction energies of polylactic(PLA)/zirconia and polylactic acid/silane/zirconia systems

\begin{tabular}{|c|c|c|c|}
\hline & & $\mathrm{E}_{\text {interaction }}(\mathrm{kca}$ & \\
\hline h k I & PLA & PLA/zirconia & PLA/silane/zirconia \\
\hline \multirow{5}{*}{$\left(\begin{array}{lll}0 & 0 & 1\end{array}\right)$} & $\alpha-L-\mathrm{LA}_{10}$ & -7.35 & -28.62 \\
\hline & $\pi-L-L_{10}$ & -9.93 & -18.13 \\
\hline & $310-L-L A_{10}$ & -13.11 & -14.94 \\
\hline & $\beta-L-\mathrm{LA}_{10}$ & -21.11 & -16.86 \\
\hline & DeSantis-LA 10 & -12.47 & -23.36 \\
\hline \multirow{5}{*}{$\left(\begin{array}{lll}1 & 0 & 0\end{array}\right)$} & $\alpha-L-L A_{10}$ & -6.67 & -19.36 \\
\hline & $\pi-L-L_{10}$ & -16.44 & -22.04 \\
\hline & $310-L-L A_{10}$ & -17.15 & -26.15 \\
\hline & $\beta-L-L A_{10}$ & -28.32 & -20.78 \\
\hline & DeSantis-LA 10 & -11.60 & -18.49 \\
\hline \multirow{5}{*}{ (1 110$)$} & $\alpha-L-L A_{10}$ & -8.96 & -15.02 \\
\hline & $\pi-L-L A_{10}$ & -13.65 & -14.94 \\
\hline & $3_{10}-L-L A_{10}$ & -9.23 & -8.12 \\
\hline & $\beta-L-\mathrm{LA}_{10}$ & -25.56 & -17.45 \\
\hline & DeSantis-LA 10 & -12.04 & -13.73 \\
\hline \multirow{5}{*}{ (1 111$)$} & $\alpha-L-L A_{10}$ & -14.21 & -22.67 \\
\hline & $\pi-L-\mathrm{LA}_{10}$ & -15.79 & -21.83 \\
\hline & $310-L-L A_{10}$ & -6.05 & -21.26 \\
\hline & $\beta-L-\mathrm{LA}_{10}$ & -18.13 & -29.00 \\
\hline & DeSantis-LA 10 & -11.69 & -24.00 \\
\hline \multirow{5}{*}{$\left(\begin{array}{lll}-1 & 0 & 0\end{array}\right)$} & $\alpha-L-L_{10}$ & -14.14 & -28.26 \\
\hline & $\pi-L-L A_{10}$ & -8.91 & -19.10 \\
\hline & $3_{10}-L-L A_{10}$ & -9.60 & -29.23 \\
\hline & $\beta-L-\mathrm{LA}_{10}$ & -23.67 & -28.28 \\
\hline & DeSantis-LA 10 & -23.56 & -18.52 \\
\hline
\end{tabular}

Table 1 suggests that the optimized $\beta$ sheets bind most strongly to the five surfaces. The interaction energies are above $20 \mathrm{kcal} / \mathrm{mol}$ except in the case of the $\left(\begin{array}{lll}1 & 1 & 1\end{array}\right)$ surface. The connections of $\alpha, \pi, 3_{10}$ optimized helices and the DeSantis structure[27] to the $\left(\begin{array}{lll}0 & 0 & 1\end{array}\right)$ surface are two-three times 
smaller than the binding of $\beta$ sheet. In the case of $\left(\begin{array}{lll}1 & 0 & 0\end{array}\right)$ surface the lowest binding energy is obtained with the $\alpha$ helix is attached to zirconia. The binding energies of $\pi$ and $3_{10}$ helices are nearly same, but with $\sim 12 \mathrm{kcal} / \mathrm{mol}$ smaller than the interaction energy of the $\beta$ sheet. The interaction energy differences between the $\beta$ structure/( $\left.\begin{array}{lll}1 & 1 & 0\end{array}\right)$ surface and the other structures/(llll 110$)$ surface are the largest. The $\alpha /\left(\begin{array}{lll}1 & 1 & 0\end{array}\right)$ zirconia and $310 /\left(\begin{array}{lll}1 & 1 & 0\end{array}\right)$ zirconia have appropriate binding energy values. The same is valid for $\pi /\left(\begin{array}{lll}1 & 1 & 0\end{array}\right)$ zirconia and

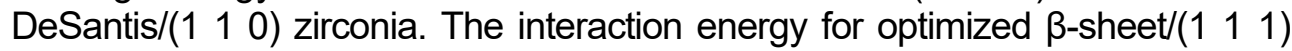
zirconia is $18 \mathrm{kcal} / \mathrm{mol}$. Three times lower than this is the interaction energy between optimized $33_{10}$ helix and $\left(\begin{array}{lll}1 & 1 & 1\end{array}\right)$ surface. The interaction energy of the optimized DeSantis structure and $(-100)$ zirconia is very close to the value resulted from $\beta$ sheet and $\left(\begin{array}{lll}-1 & 0 & 0\end{array}\right)$ interaction. The energy difference toward the less stable system is $14 \mathrm{kcal} / \mathrm{mol}$.

Table 1 also shows that the coupling agents generally increase the interaction energies. The most striking increases were observed for optimized $\alpha$ and $3_{10}$ helices on zirconia surfaces. The interaction energies increase about three-four times in the case of optimized $\alpha$ helix/(llll $\left.\begin{array}{lll}0 & 1\end{array}\right)$ surface, $\alpha$ helix/(llll $\left.\begin{array}{ll}0 & 0\end{array}\right)$

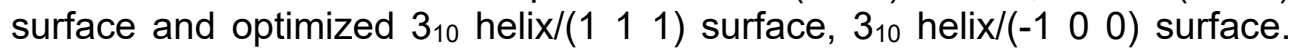
However, the silanes do not always increase the interaction energies - as ween e.g. in the $\beta$ structure and $\left(\begin{array}{lll}0 & 0 & 1\end{array}\right),\left(\begin{array}{lll}1 & 0 & 0\end{array}\right),\left(\begin{array}{lll}1 & 1 & 0\end{array}\right)$ surface systems.

The top layers of the $\left(\begin{array}{lll}0 & 0 & 1\end{array}\right),\left(\begin{array}{lll}1 & 0 & 0\end{array}\right),\left(\begin{array}{lll}1 & 1 & 1\end{array}\right)$ and $\left(\begin{array}{lll}-1 & 0 & 0\end{array}\right)$ zirconia surfaces are constituted by zircon atoms. In contrast the oxygen atoms are on the interface of the bioceramic (1 110$)$ surface. The polymer chains and the zirconia surfaces are held together by weak interactions. These interactions occur between the hydrogen atoms from methyl groups or from the main chains of the polymer and the oxygen atoms of the surfaces. Another attraction is formed between the carbonyl oxygen from the polylactic acid and the zirconium atoms of the bioceramic. These interactions influence the behavior of the polymer chains. The conformation changes after the simulations can be seen in Figure 1. In most cases the polymers remain parallel to the surfaces, but they change their positions compared to the reference surfaces. The conformations of the polymers undergo major changes when silane coupling agents are laid between the zirconia surfaces and the polylactic acids (Figure 2). These changes are influenced by interactions between the silane molecules and the polymers. Interactions occur between hydrogen atoms from methyl groups or from main chains of the polymers and the hydroxyl oxygens of the silane molecules. Another type of interaction takes place between the carbonyl or carboxyl oxygen atom of the polymer and the hydrogen atom of the silane molecule. 


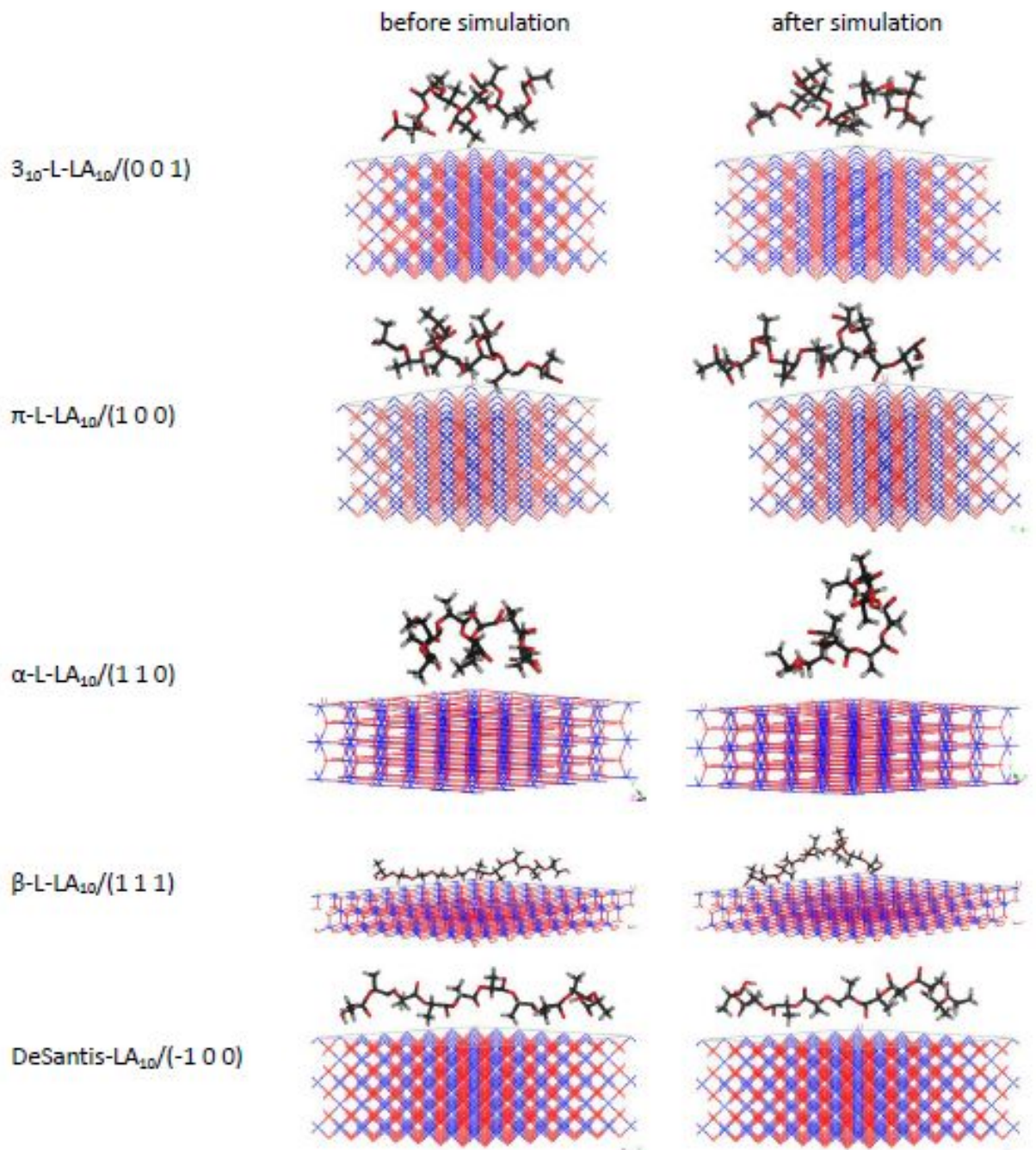

Figure 1. Snapshots of PLA on zirconia surfaces before and after the molecular dynamics simulations

Table 2 shows the interaction energies of optimized PLA models and hydroxyapatite systems with and without coupling agents. The energy values are much smaller than in the polylactic acid/zirconia systems, nut still generally favorable - with one exception: the optimized a helix did not attach to the (1 111 ) hydroxyapatite surface. Overall, the interaction energies for PLA in Table 1 are relatively small and essentially degenerate - implying that no single type of secondary structure would be favored in these interactions. 
The optimized $\pi$ and $\beta$ decameric units are hardly linked to $\left(\begin{array}{lll}0 & 0 & 1\end{array}\right)$ and $\left(\begin{array}{lll}1 & 1 & 0\end{array}\right)$ surfaces, respectively. The optimized DeSantis structure binds to $\left(\begin{array}{lll}0 & 0 & 1\end{array}\right),\left(\begin{array}{ll}1 & 1\end{array}\right)$, $\left(\begin{array}{lll}1 & 1 & 1\end{array}\right)$ and $\left(\begin{array}{lll}-1 & 0 & 0\end{array}\right)$ hydroxyapatites with interaction energies above $4 \mathrm{kcal} / \mathrm{mol}$. 3-6 kcal/mol interaction energies exist between optimized $\pi$ helices and $\left(\begin{array}{lll}1 & 1 & 0\end{array}\right)$, $\left(\begin{array}{lll}1 & 1 & 1\end{array}\right)$ and $\left(\begin{array}{lll}-1 & 0 & 0\end{array}\right)$ surfaces. The interface energies are around $4-5 \mathrm{kcal} / \mathrm{mol}$ when the $33_{10}$ structures are connected to $\left(\begin{array}{lll}1 & 1 & 0\end{array}\right)$ and $\left(\begin{array}{lll}-1 & 0 & 0\end{array}\right)$ hydroxyapatite surfaces.

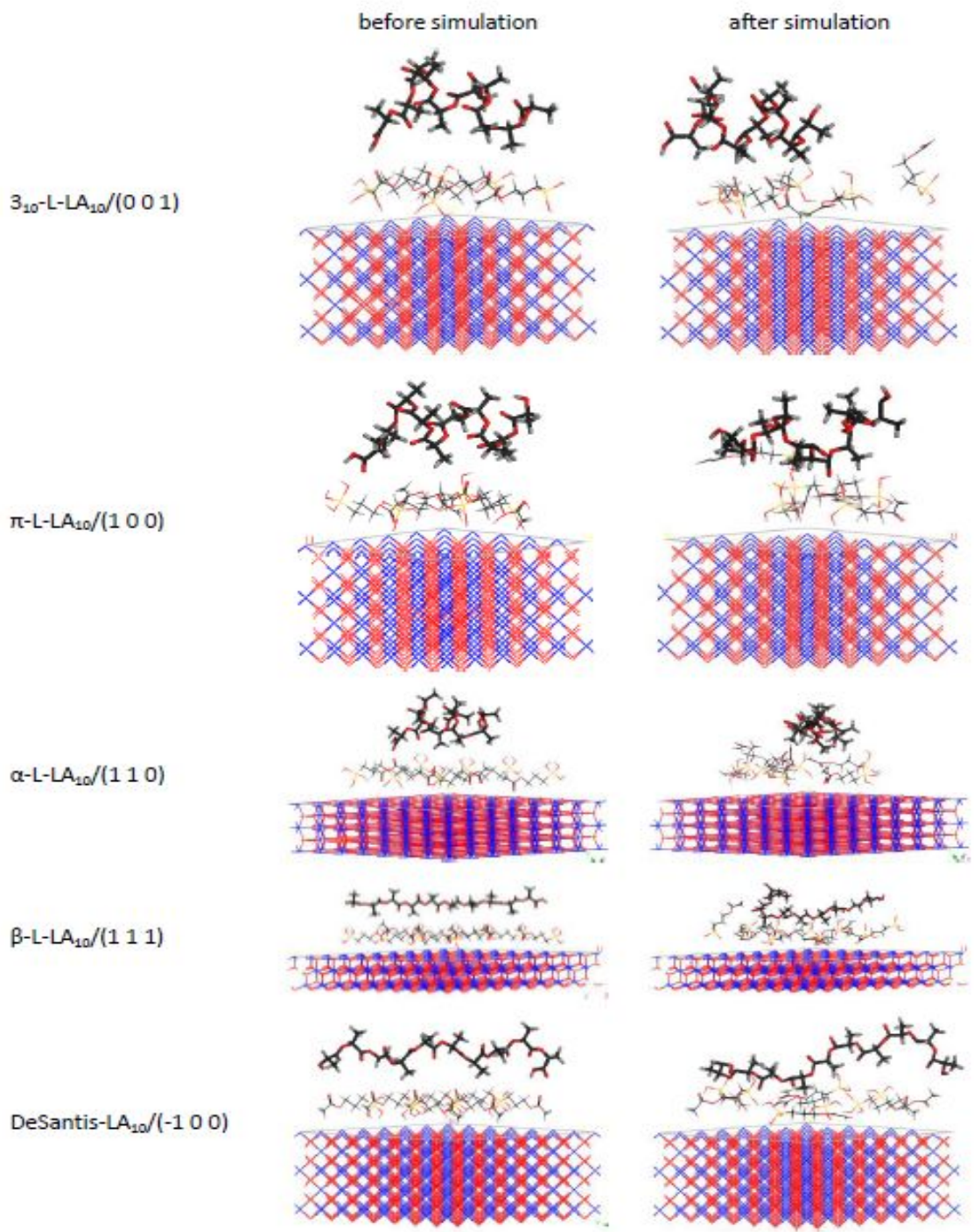

Figure 2. Snapshots of PLA on silane/zirconia surfaces before and after the simulations 
Table 2. The interaction energies of polylactic (PLA)/hydroxyapatite (HA) and polylactic acid/silane/hydroxyapatite systems

\begin{tabular}{|c|c|c|c|}
\hline \multirow[b]{2}{*}{ h k I } & \multirow[b]{2}{*}{ PLA } & \multicolumn{2}{|c|}{$E_{\text {interaction }}(\mathrm{kcal} / \mathrm{mol})$} \\
\hline & & PLA/HA & PLA/silane/HA \\
\hline \multirow{5}{*}{$\left(\begin{array}{lll}0 & 0 & 1\end{array}\right)$} & $\alpha-L-L_{10}$ & -0.79 & -17.46 \\
\hline & $\pi-L-L A_{10}$ & -0.07 & -11.44 \\
\hline & $3_{10}-L-L A_{10}$ & -1.52 & -22.67 \\
\hline & $\beta-L-L A_{10}$ & -1.95 & -15.77 \\
\hline & DeSantis-LA 10 & -3.90 & -31.63 \\
\hline \multirow{5}{*}{$\left(\begin{array}{lll}1 & 0 & 0\end{array}\right)$} & $\alpha-L-L A_{10}$ & -1.50 & -11.78 \\
\hline & $\pi-L-L_{10}$ & -1.13 & -18.54 \\
\hline & $3_{10}-L-L A_{10}$ & -2.16 & -10.57 \\
\hline & $\beta-L-L A_{10}$ & -2.60 & -29.59 \\
\hline & DeSantis-LA 10 & -0.47 & -12.48 \\
\hline \multirow{5}{*}{$\left(\begin{array}{lll}1 & 1 & 0\end{array}\right)$} & $\alpha-L-L A_{10}$ & -0.84 & -9.64 \\
\hline & $\pi-L-L_{10}$ & -4.76 & -12.05 \\
\hline & $3_{10}-\mathrm{L}-\mathrm{LA}_{10}$ & -4.96 & -0.36 \\
\hline & $\beta-L-L A_{10}$ & -0.18 & -8.32 \\
\hline & DeSantis-LA 10 & -6.63 & -12.74 \\
\hline \multirow{5}{*}{$\left(\begin{array}{lll}1 & 1 & 1\end{array}\right)$} & $\alpha-L-L A_{10}$ & 0.46 & -12.33 \\
\hline & $\pi-L-L A_{10}$ & -3.13 & -8.60 \\
\hline & $3_{10}-\mathrm{L}-\mathrm{LA}_{10}$ & -1.24 & -7.80 \\
\hline & $\beta-L-L A_{10}$ & -2.74 & -17.27 \\
\hline & DeSantis-LA 10 & -6.16 & -9.20 \\
\hline \multirow{5}{*}{$\left(\begin{array}{lll}-1 & 0 & 0\end{array}\right)$} & $\alpha-L-L A_{10}$ & -1.84 & -15.68 \\
\hline & $\pi-L-L_{10}$ & -5.98 & -4.30 \\
\hline & $3_{10}-L-L A_{10}$ & -3.99 & -15.90 \\
\hline & $\beta-L-L A_{10}$ & -3.68 & -27.32 \\
\hline & DeSantis-LA 10 & -5.41 & -15.26 \\
\hline
\end{tabular}

In most cases seen in Table 2, silanes have a much larger effect on the PLA/hydroxyapatite interactions than in the case of zirconia. Thus, the interaction energy of optimized $\pi$ helix increases by two orders of magnitude with the addition of coupling agent. However the interaction energy decrease when the helix is attached to $(-100)$ hydroxyapatite in the presence of silane. 
The interactions energies between optimized $\beta$ sheets and the hydroxyapatite surfaces increase in all cases with the addition of silanes.

Weak interactions hold together PLA and hydroxyapatite systems. The general formula of hydroxyapatite is $\mathrm{Ca}_{10}\left(\mathrm{PO}_{4}\right)_{6}(\mathrm{OH})_{2}$. The top layers of the surfaces have different atom compositions by cleaving the crystal along planes. Oxygen, calcium and phosphorus atoms are at the $\left(\begin{array}{lll}1 & 0 & 0\end{array}\right),\left(\begin{array}{lll}1 & 1 & 0\end{array}\right)$ and $\left(\begin{array}{lll}-1 & 0 & 0\end{array}\right)$ surfaces. The interface of $\left(\begin{array}{lll}0 & 0 & 1\end{array}\right)$ hydroxyapatite contains oxygen and hydrogen molecules. Oxygen, hydrogen and phosphorus atoms constitute the top layers of $\left(\begin{array}{lll}1 & 1 & 1\end{array}\right)$ surface. The PLA/apatite interactions entail the hydrogen atoms from methyl groups or from the main chains of PLA and the oxygens of the surfaces. Interactions exist between oxygen from ester groups of PLA and hydroxyl groups in hydroxyapatite. PLA change their conformations after molecular dynamic simulations due to the interactions. The polymers did not remain parallel with the surface (Figure 3 ). Interactions between the polylactic acids and silane molecules exist when coupling agents are added to the system. The conformations of the decameric units were not preserved in these cases neither (Figure 4).

\section{CONCLUSIONS}

Molecular dynamics simulations were used to analyze the interfacial behaviors of polylactic acids and zirconia, hydroxyapatite surfaces. The interactions of polymers on five crystallographic planes were simulated. The interaction energies between the DFT optimized polymers and bioceramic surfaces was analyzed. The polylactic acids bind to the polymers in every situation. There is one exception, optimized $\alpha$ helix did not attach to the $\left(\begin{array}{lll}1 & 1 & 1\end{array}\right)$ hydroxyapatite surface. Optimized $\beta$ sheets bind most strongly to the five zirconia surfaces, the interaction energies are above $20 \mathrm{kcal} / \mathrm{mol}$. The energy values are much smaller in polylactic acid/hydroxyapatite systems than in the polylactic acid/zirconia systems. The silane coupling agents can improve the interactions between the bioceramic surfaces and the polylactic acids.

The effects of the coupling agents are more evident if the surface is hydroxyapatite.

Weak interactions hold together the polylactic acids and bioceramic systems. These interactions are formed between the hydrogen atoms from methyl groups or from the main chains of the polylactic acids and the oxygens of the surfaces. Polylactic acids change their conformations after molecular dynamics simulations due to the interactions. The conformation changes are more obvious when silane coupling agents are added to the polylactic acids and bioceramic systems. 
POLYLACTIC ACID INTERACTIONS WITH BIOCERAMIC SURFACES

before simulation

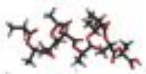

$\alpha-L-L A_{10} /\left(\begin{array}{llll}0 & 0 & 1\end{array}\right)$

$\beta-L-L A_{10} /\left(\begin{array}{lll}1 & 0 & 0\end{array}\right)$

DeSantis-LA $10 /(110)$

$\pi-L-L A_{10} /\left(\begin{array}{lll}1 & 1 & 1\end{array}\right)$

$3_{10}-L-L A_{10} /\left(\begin{array}{lll}-1 & 0 & 0\end{array}\right)$
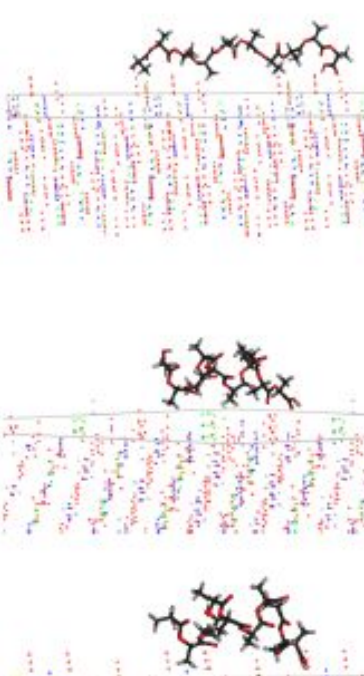

after simulation

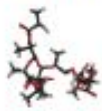

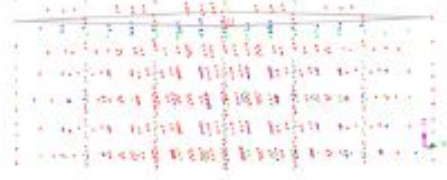
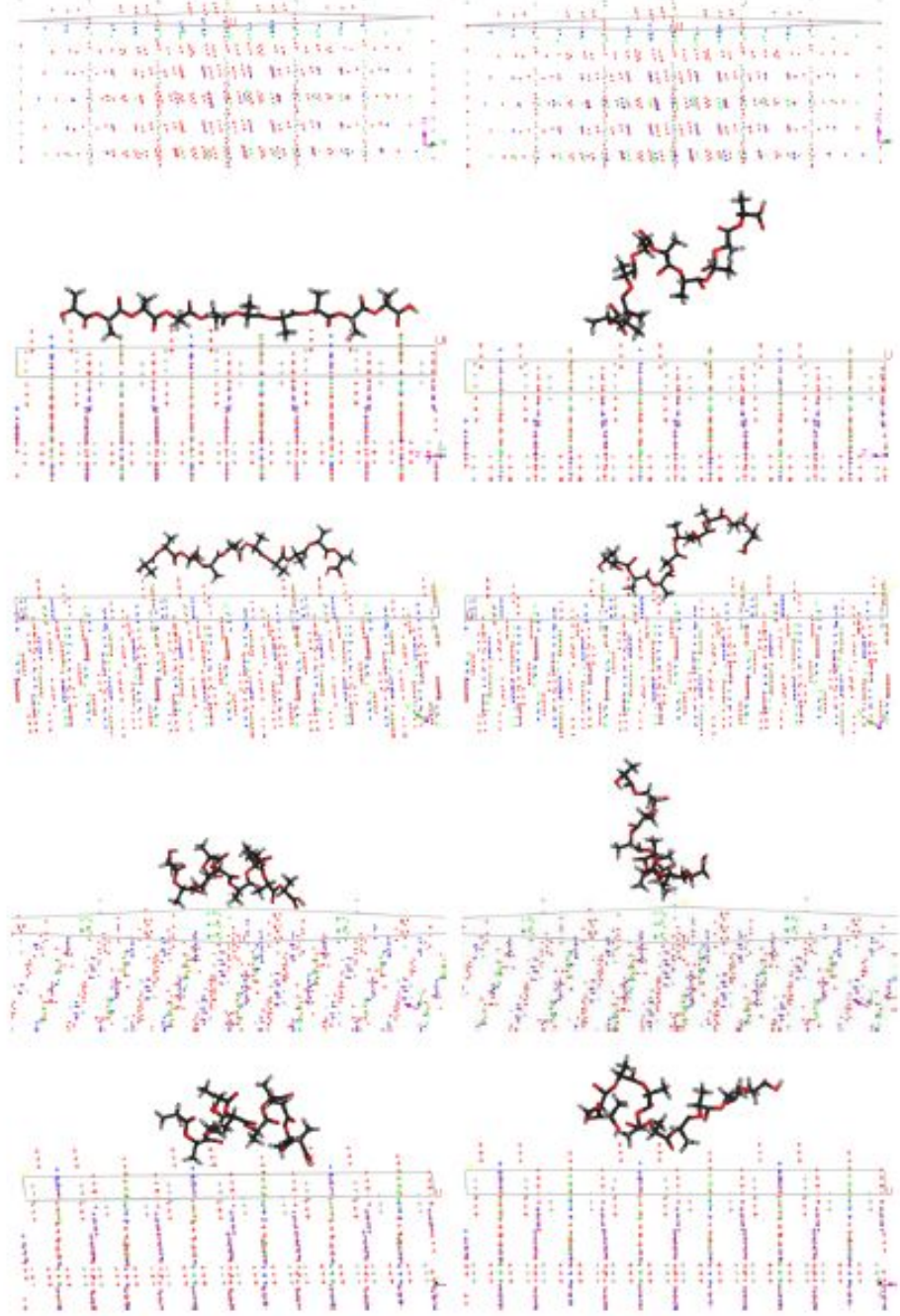

Figure 3. Snapshots of polylactic acids on hydroxyapatite surfaces before and after the simulations 
$\alpha-L-L A_{10} /\left(\begin{array}{lll}0 & 0 & 1\end{array}\right)$

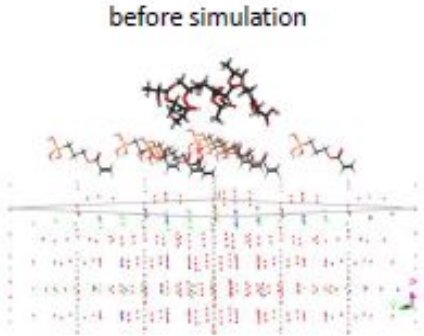

after simulation

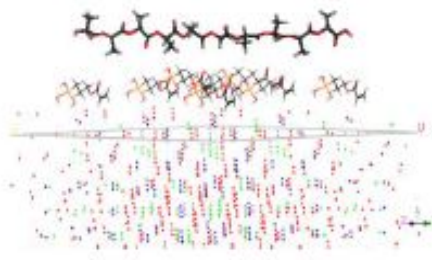

$\beta-L-L A_{10} /\left(\begin{array}{llll}1 & 0 & 0\end{array}\right)$

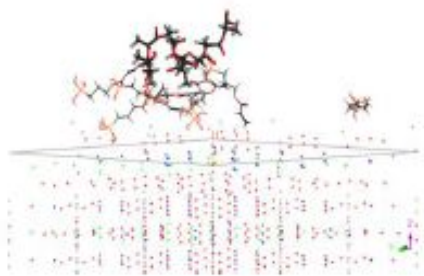

$\beta-L-L A_{10} /(100)$

DeSantis-LA $10 /\left(\begin{array}{lll}1 & 1 & 0\end{array}\right)$
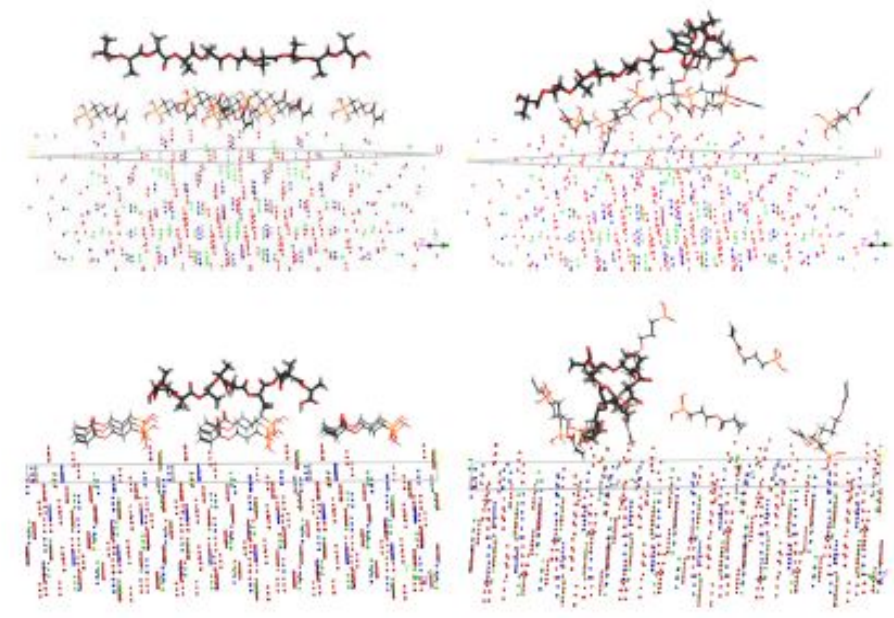

$\pi-L-L A_{10} /\left(\begin{array}{lll}1 & 1 & 1\end{array}\right)$

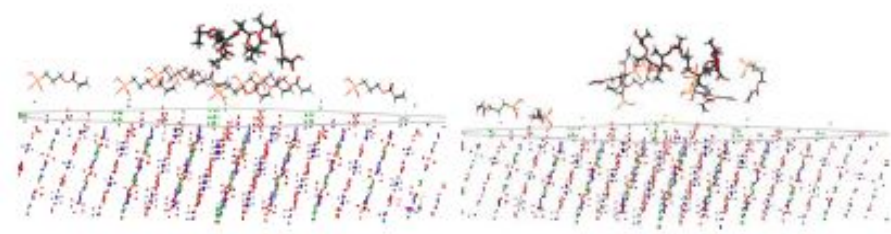

$3_{10}-L-L A_{10} /(-100)$
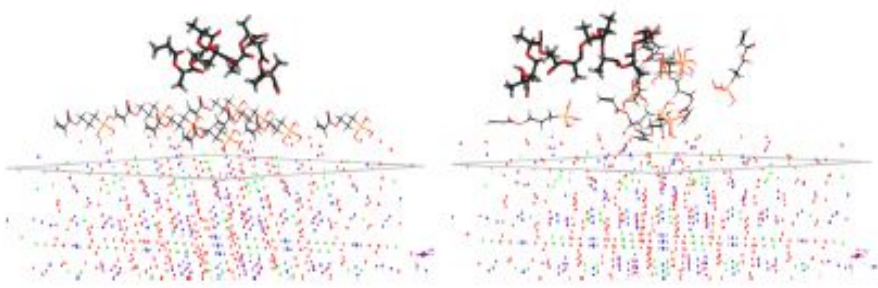

Figure 4. Snapshots of polylactic acids on silane/hydroxyapatite surfaces before and after the simulations 


\section{EXPERIMENTAL SECTION}

\section{Models of polymer molecules}

Five PLA decameric units were employed throughout this study. The secondary-type structures ( $\alpha, \pi, 3_{10}, \beta$, DeSantis [27]) were optimized with at the $M 062 x / 6-311+G^{* *}$ level of theory [28] as previously described $[29,30]$.

\section{Models of bioceramic surfaces}

The two bioceramics surfaces utilized in this study were hydroxyapatite and zirconia. The surfaces were cleaved along (lll $\left.0 \begin{array}{lll}0 & 1\end{array}\right),\left(\begin{array}{lll}1 & 0 & 0\end{array}\right),\left(\begin{array}{lll}1 & 1 & 0\end{array}\right),\left(\begin{array}{lll}1 & 1 & 1\end{array}\right)$ and $\left(\begin{array}{lll}-1 & 0 & 0\end{array}\right)$ planes, followed by minimizations under boundary conditions with a non-bond cut-off distance of $9.5 \AA$. The zirconia was created using built-in options of Material Studio software for creating ceramics (cf. Table 3). The hydroxyapatite surface was constructed based on crystallographic data (cf. Table 4) [31].

\section{PLA/bioceramics interactions}

The PLA/bioceramics models were constructed by placing the polymer chains on the surface. Molecular dynamic simulations were conducted on PLA/bioceramics interfacial model under NVT ensemble with a time step of $1 \mathrm{fs}$ for $5 \mathrm{ps}$. The binding energies between the PLA and the surfaces were calculated after molecular dynamic simulations.

\section{PLA/coupling agents/bioceramics interactions}

Silane (3 acryloxypropyltrihydroxysilane) was used to investigate the effects of the coupling agents on the binding energies between the polylactic acid and bioceramics. The coupling agent models were built on all cleaved surfaces. 6-9 silane molecules were randomly distributed on the bioceramics surface. Geometry optimizations were performed on the surfaces /coupling agent systems. Then polylactic acid chains were placed on the systems. The molecular dynamic simulations were also conducted under NVT ensemble at $300 \mathrm{~K}$ temperature for $5 \mathrm{ps}$.

The adhesion between the polylactic acid and bioceramic surfaces can be evaluated by the interaction energy between them. The interaction energies were calculated through the following equation:

$$
E_{\text {interaction }}=E_{\text {surface+polymer }}-\left(E_{\text {surface }}+E_{\text {polymer }}\right)
$$

where $E_{\text {surface+polymer }}$ is the energy of the surface with PLA polymer, $E_{\text {surface }}$ is the energy of the surface and $E_{\text {polymer }}$ is the energy of the polymer. The binding energy is obtained by dividing the interaction energy to the number of monomers existing in the polymers. The high binding energy suggests high adhesive strength between the surface and the polymer. 
The coupling agents increase molecular binding between bioceramics and polymers. The interaction energies in surface/coupling agent/polymer system:

$$
E_{\text {interaction }}=E_{\text {surface+coupling agent+polymer }}-\left(E_{\text {surface+coupling agent }}+E_{\text {polymer }}\right)
$$

where $E_{\text {surface+coupling agent+polymer is the energy of the surface with silane and }}$ PLA polymer, $E_{\text {surface+coupling agent }}$ is the energy of the surface with silane and $E_{\text {polymer }}$ is the energy of the polymer.

The molecular dynamics simulations were conducted using UFF force field [32] as implemented in the Forcite module of Materials Studio package [33]. The potential energy is expressed as a sum of valence or bonded interactions and non-bonded interactions.

$$
\mathrm{E}=\mathrm{E}_{\mathrm{R}}+\mathrm{E}_{\theta}+\mathrm{E}_{\Phi}+\mathrm{E}_{\omega}+\mathrm{E}_{\mathrm{vdW}}+\mathrm{E}_{\mathrm{el}}
$$

The valence interactions consist of bond stretching $\left(E_{R}\right)$ and angular distortions (Equation 3). Angular distortions include angle bending $\left(\mathrm{E}_{\theta}\right)$, dihedral angle torsion $\left(E_{\phi}\right)$ and inversion terms $\left(E_{\omega}\right)$. The non-bonded interactions involve van der Waals term ( $\left.E_{\mathrm{vdW}}\right)$ and electrostatic term $\left(\mathrm{E}_{\mathrm{el}}\right)$.

Table 3. Models of zirconia surfaces. Color codes: blue represent zirconium atom and red the oxygen atom.

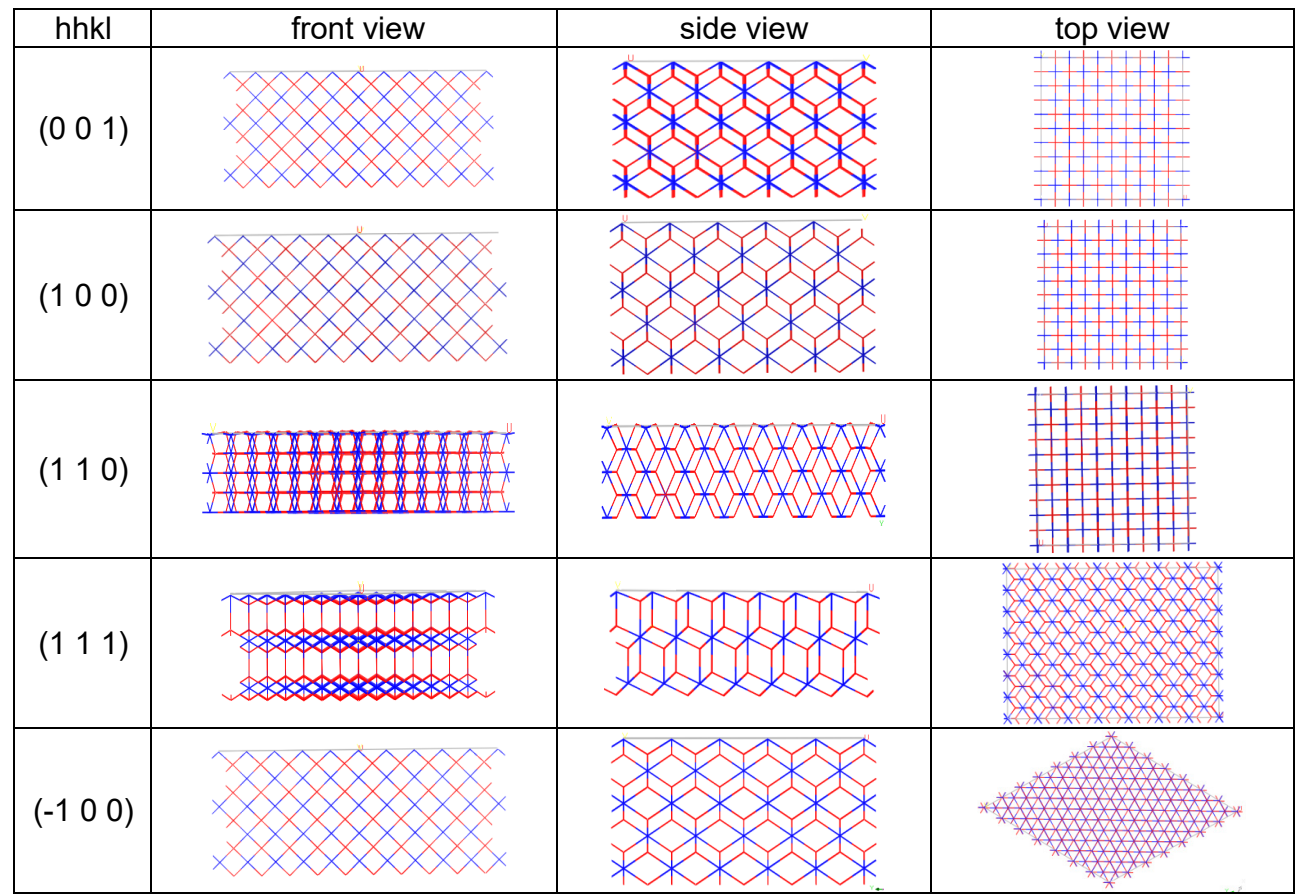


Table 4. Models of hydroxyapatite surfaces. Color codes: blue represent calcium, red oxygen, green phosphorus atom and gray hydrogen atoms

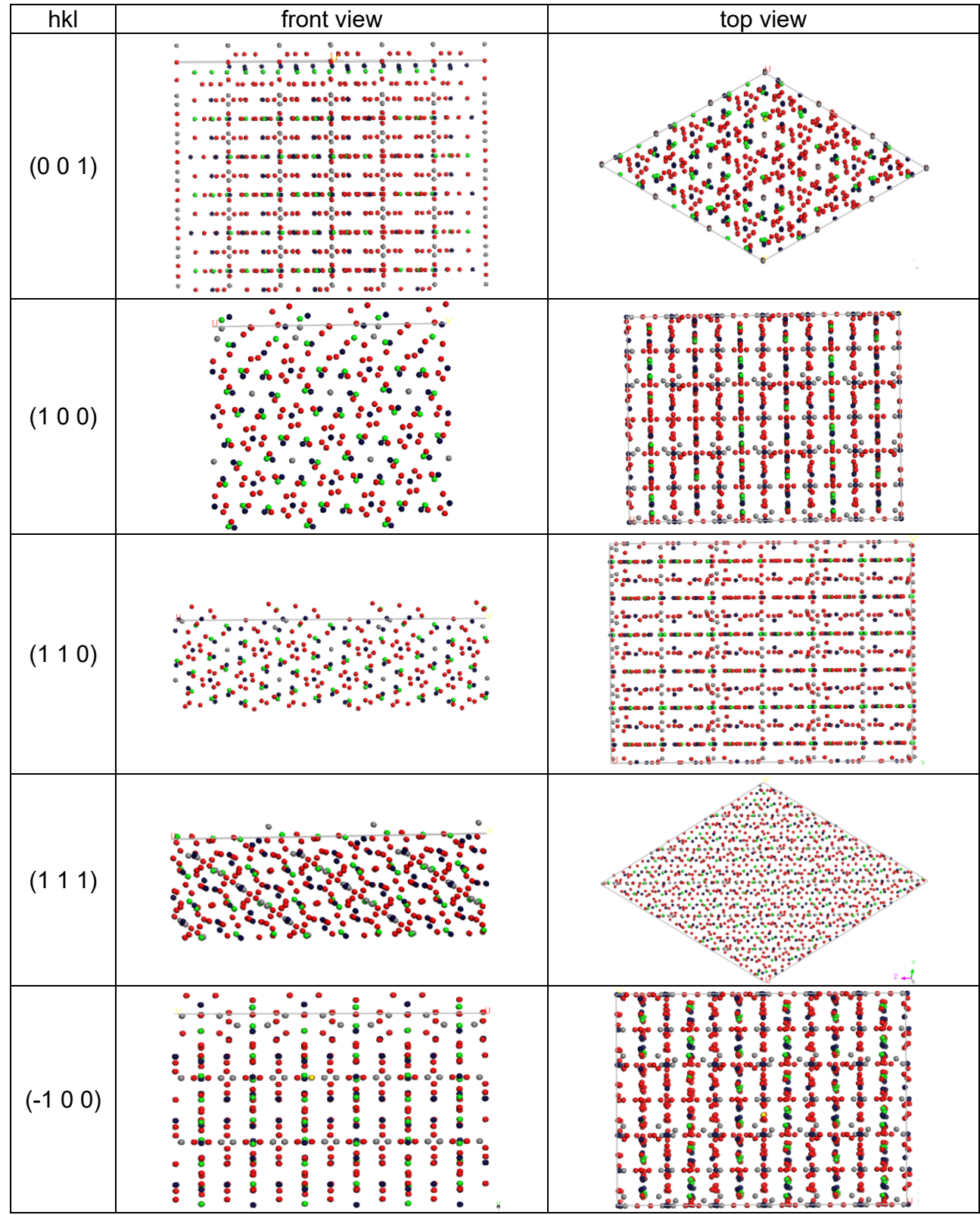


Molecular dynamics simulations were employed to study polylactic $\mathrm{acid} /$ bioceramics interface interactions. The study analyzed the binding energies between polylactic acid and two bioceramics: hydroxyapatite and zirconia. The interactions of polylactic acid on the surfaces crystallographic planes $\left(\begin{array}{lll}0 & 0 & 1\end{array}\right)$, $\left(\begin{array}{lll}1 & 0 & 0\end{array}\right),\left(\begin{array}{lll}1 & 1 & 0\end{array}\right),\left(\begin{array}{lll}1 & 1 & 1\end{array}\right)$ and $\left(\begin{array}{lll}-1 & 0 & 0\end{array}\right)$ were simulated. The effects of coupling agents on interfacial binding energies were also examined.

\section{ACKNOWLEDGMENTS}

Funding from the Romanian Ministry of Education and Research (PN-IIIP22.1-PED2019-2293) and from the Babes-Bolyai University (to BAMV) is gratefully acknowledged.

\section{REFERENCES}

1. B. D. Ratner; A. S. Hoffman; F. J. Schoen; J. E. Lemons; Biomaterials Science, An Introduction to Materials in Medicine (Elsevier Academic Press, 2004).

2. P. F. Manicone; P. Rossi lommetti; L. Raffaelli; J. Dent. 2007, 35, 819-826.

3. J. R. Piascik; S. D. Wolter; B. R. Stoner; Dent. Mater. 2011, 27, e99-e105.

4. A. Attia; F. Lehmann; M. Kern; Dent. Mater. 2011, 27, 207-213.

5. R. L. Smith; C. Villanueva; J. K. Rothrock; C. E. Garcia-Godoy; B. R. Stoner; J. R. Piascik; J. Y. Thompson; Dent. Mater. 2011, 27, 779-785.

6. R. Amaral; M. Özcan; M. A. Bottino; L. F. Valandro; Dent. Mater. 2006, 22, 283-290.

7. S. Kitayama; T. Nikaido; R. Takahashi; L. Zhu; M. Ikeda; R. M. Foxton; A. Sadr; J. Tagami; Dent. Mater. 2010, 26, 426-432.

8. M. N. Aboushelib; H. Mirmohamadi; J. P. Matinlinna; E. Kukk; H. F. Ounsi; Z. Salameh; Dent. Mater. 2009, 25, 989-993.

9. J. P. Matinlinna; L. V. J. Lassila; P. K. Vallittu; J. Dent. 2006, 34, 740-746.

10. J. R. Piascik; E. J. Swift; J. Y. Thompson; S. Grego; B. R. Stoner; Dent. Mater. 2009, 25, 1116-1121.

11. J. P. Matinlinna; T. Heikkinen; M. Özcan; L. V. J. Lassila; P. K. Vallittu; Dent. Mater. 2006, 22, 824-831.

12. R. Di Maggio; S. Dirè; E. Callone; F. Girardi; G. Kickelbick; Polymer (Guildf). 2010, 51, 832-841.

13. R. P. Singh; J. D. Way; S. F. Dec; J. Memb. Sci. 2005, 259, 34-46.

14. J. Han; C. Zuo; Q. Gu; D. Li; X. Wang; G. Xue; Appl. Surf. Sci. 2008, 255, 2316-2321.

15. A. Casucci; E. Osorio; R. Osorio; F. Monticelli; M. Toledano; C. Mazzitelli; M. Ferrari; J. Dent. 2009, 37, 891-897.

16. J. F. Mano; R. A. Sousa; L. F. Boesel; N. M. Neves; R. L. Reis; Compos. Sci. Technol. 2004, 64, 789-817.

17. M. Darder; P. Aranda; E. Ruiz-Hitzky; Adv. Mater. 2007, 19, 1309-1319. 
18. R. Murugan; S. Ramakrishna; Compos. Sci. Technol. 2005, 65, 2385-2406.

19. J. Russias; E. Saiz; R. K. Nalla; K. Gryn; R. O. Ritchie; A. P. Tomsia; Mater. Sci. Eng. C 2006, 26, 1289-1295.

20. L. Fang; Y. Leng; P. Gao; Biomaterials 2006, 27, 3701-3707.

21. E. Smolko; G. Romero; Radiat. Phys. Chem. 2007, 76, 1414-1418.

22. X. Zhang; Y. Li; G. Lv; Y. Zuo; Y. Mu; Polym. Degrad. Stab. 2006, 91, 12021207.

23. Y. Zuo; Y. Li; J. Li; X. Zhang; H. Liao; Y. Wang; W. Yang; Mater. Sci. Eng. A 2007, 452-453, 512-517.

24. M. Todo; S. D. Park; K. Arakawa; Y. Takenoshita; Compos. Part A Appl. Sci. Manuf. 2006, 37, 2221-2225.

25. H. ping Zhang; X. Lu; Y. Leng; L. Fang; S. Qu; B. Feng; J. Weng; J. Wang; Acta Biomater. 2009, 5, 1169-1181.

26. L. M. Mathieu; T. L. Mueller; P. E. Bourban; D. P. Pioletti; R. Müller; J. A. E. Månson; Biomaterials 2006, 27, 905-916.

27. P. . De Santis; J. Kocacs; 1968, 6, 299-306.

28. Y. Zhao; D. G. Truhlar; Theor. Chem. Acc. 2008, 120, 215-241.

29. I. Irsai; A. Lupan; C. Majdik; R. Silaghi-Dumitrescu; Stud. Univ. Babes-Bolyai Chem. 2017, 62, 495-513.

30. I. Irsai; C. Majdik; A. Lupan; R. Silaghi-Dumitrescu; J. Math. Chem. 2012, 50, 703-733.

31. R. M. Wilson; J. C. Elliott; S. E. P. Dowker; Am. Mineral. 1999, 84, 1406-1414.

32. A. K. Rappe; C. J. Casewit; K. S. Colwell; W. A. Goddard III; W. M. Skiff; J. Am. Chem. Soc. 1992, 114, 10024-10035.

33. 2017 Dassault Systèmes BIOVIA, Materials Studio, 2017, San Diego: Dassault Systèmes. 
